\title{
RETRACTED ARTICLE: Self-calibration of redundandly actuated PKM via motion reverse points: optimization procedure
}

\author{
Maurizio Ruggiu
}

Received: 1 April 2015/Accepted: 5 November 2015/Published online: 13 November 2015

(C) Springer Science+Business Media Dordrecht 2015

This article has been retracted by the author and the Editor-in-Chief per the Committee on Publication Ethics (COPE) guidelines on redundant publication. The article shows an overlap with the following sources: Latest Advances in Robot Kinematics, Springer 2012, ISBN 978-94-007-4619-0 Compu- tational Kinematics, Proceedings of the 6th International Workshop on Computational Kinematics (CK2013), Springer 2014, ISBN 978-94-007-7213-7 The online version of this article contains the full text of the retracted article as electronic supplementary material.

Electronic supplementary material The online version of this article (doi:10.1007/s11012-015-0321-y) contains supplementary material, which is available to authorized users.

M. Ruggiu ( $\square)$

Department of Mechanical, Chemical and Materials

Engineering, University of Cagliari, Cagliari, Italy

e-mail: maurizio.ruggiu@dimcm.unica.it 